\title{
ARTICLE
}

\section{A Hybrid Regression Neural Network Model for Assessment of Alpha Particle Activity Channels using LRAD}

\author{
Mingzhe LIU ${ }^{1,2}$, Xianguo TUO ${ }^{1,2}$, Jiansheng $\mathrm{Wu}^{3}, \mathrm{Zhe} \mathrm{Li}^{2}$, Lei WANG ${ }^{2}$ \\ ${ }^{1}$ State Key Laboratory of Geohazard Prevention and Geoenvironment Protection, Chengdu University of Technology, Chengdu 610059, \\ China \\ ${ }^{2}$ College of Nuclear Technology and Automation Engineering, Chengdu University of Technology, Chengdu 610059, China \\ ${ }^{3}$ School of Information Engineering, Wuhan University of Technology, Wuhan 430070, China
}

\begin{abstract}
Long Range Alpha Detectors (LRAD) have been recently used to measure alpha particles emitting contamination inside decommissioned channels. We studied the relation between six parameters (channel diameter, channel length, distance to radioactive source, radioactive strength, wind speed and flux) for LRAD measurement and the data on ionizing current. We found these parameters showing complex linear and nonlinear relationships to measuring results. To achieve the activity correction of alpha particles, a hybrid regression neural network approach is proposed. In this model, two linear regression models are used to capture linear characteristics while four nonlinear regression models based on neural networks are for nonlinear characteristics. Then the hybrid parametric regression approach is used for ensemble of these models based on the principal component analysis. The comparison of computational results of the approach with normal regression models confirms its clear advantage for dealing with this complex nonlinear correction. Thus, an integrated picture of alpha particle activity inside contaminated channels can be obtained.
\end{abstract}

\section{KEY WORDS: regression, neural network, alpha, activity assessment, LRAD}

\section{Introduction}

With the rapid development of nuclear industry over the last 50 years, nuclear decommissioning has become the hot topic within the industry worldwide. Most nuclear facility dismantling is involved in contaminated channel disassembling. Recently, a Long Range Alpha Detector (LRAD) technique has been used to measure alpha particles emitting contamination inside channels1)-7). LRAD measures ion charged produced by alpha particles in air, and has various advantages over conventional techniques1)-7). This technique provides an alternative method to measure alpha emitting contamination on internal surfaces of contaminated channels. When air is exposed to alpha radiation, charged ions are generated. The detector system collects the ions and counts the number of ions produced by the radiation. The number of ions is proportional to the amount of emitting alpha particles on the monitored surface. Thus the system can measure contamination on internal surfaces of channels.

Normally, the result of a LRAD measurement depends on six parameters, i.e., channel diameter, channel length, distance to radioactive source, radioactive source strength, wind speed and flux. Our statistical analysis to LRAD measurement results indicates that the distance to (radioactive) source and the (radioactive) source strength approximately show linear relationships to ion current while the rest parameters show nonlinear correspondence (to ion current). That means, there is a complex relationship

*Corresponding author, E-mail: liumz@cdut.edu.cn

(C) 2012 Atomic Energy Society of Japan, All Rights Reserved. between the parameter space and measuring results. Therefore, a nonlinear method should be used to deal with the LRAD data. To our best knowledge, this issue has been little studied using a hybrid method considering both linear and nonlinear characteristics so far.

Artificial neural networks (ANN) have been recognized as more efficient models than conventional statistical forecasting ones for solving nonlinear issues. With regard to conventional methods, it is very difficult to find an appropriate mathematical function to describe this kind of complex nonlinear relationship in LRAD measurement. A great number of ANN models have been presented and applied to various fields in solving nonlinear issues from natural to man-made systems. Currently, hybrid forecasting models have also been developed to improve prediction accuracy. Bowden et al. presented two methodologies, namely partial mutual information and self-organizing map integrated with a genetic algorithm and general regression neural network [8-9]. Ashu et al. applied a hybrid neural network model for hydrologic time series forecasting [10]. Some recent studies clearly demonstrate that the hybrid model could provide an effective way to improve the forecasting accuracy achieved by either of the models used separately.

Although hybrid ANN models have found many applications in a variety of areas, study of hybrid ANN models is little involved in the cases containing both linear and nonlinear patterns, especially, in the complex nuclear data processing. Based on excellent ANN research work in the literature and our experiments using the LRAD, this paper presents a hybrid regression NN (HRNN) model for 
alpha particles activity assessment. The computational results of the HRNN approach have shown its clear advantage for dealing with this complex nonlinear problem, compared to normal regression ensemble models.

This paper is organized as follows. In Section 2, linear and nonlinear regression models are presented to describe linear and nonlinear characteristics in the measurement system. The hybrid regression ensemble model is proposed to integrate the linear and nonlinear output. Section 3 analyzes the results of LRAD experiments using the HRNN model, and compares with measured values. The conclusion is given in Section 4.

\section{Hybrid NN Ensemble Model}

\section{Flow Chart of Hybrid Regression NN Model}

The proposed hybrid regression NN model includes the following steps: (1) two different linear regression models are used to get linear forecasting output; (2) four nonlinear regression models are adopted for nonlinear forecasting output; (3) the principal component analysis (PCA) method is used to extract ensemble members from linear and nonlinear forecasting output; (4) the proposed HRNN model is then used to combine the selected individual forecasting results into a hybrid parametric ensemble model.

\section{Linear and Nonlinear Regression Models}

To take account of both linear and nonlinear characteristics of independent variable, in LRAD experiments for channels, we adopt two different linear regression models, i.e., partial least squares (PLS) regression [11-12] and multi recursive regression (MRR) [13] to capture the linear characteristics of this complex measurement system. With regard to nonlinear characteristics, four models including multi-layer perceptions based on the Levenberg-Marquard algorithm (MLP), the radial basis function network (RBF), and 2 general regression neural networks (GRNNs) are used to describe the nonlinear patterns in the system.

\section{Hybrid Regression NN Ensemble Model}

The hybrid parametric regression (also known as semi-parametric regression) models have been widely applied to many fields such as economics and medical science14), and hybrid parametric regression is an emerging field that fuses traditional parametric regression and nonparametric regression methods.

Suppose yi is the independent variable, $\mathrm{xi}$ is the $\mathrm{m}$ vector of covariates and zi is an unknown centered smooth function. The output yi depends on xi and zi through the following semi-parametric regression model

$$
\mathrm{yi}=\mathrm{xiT} \beta+\mathrm{h}(\mathrm{zi})+\varepsilon \mathrm{i}
$$

where $\beta$ is a $m$ vector of regression coefficients, and the errors $\varepsilon_{i}$ are assumed to be independent and follow $N\left(0, \sigma^{2}\right)$. $x_{i}^{T} \beta$ is the parametrical part of model for epitaxial forecasting, and its objective is to control the independent variable trend. $h\left(z_{i}\right)$ is the non-parametrical part of model for local adjustment so that it is better to fit response value. A solution can be obtained by minimizing the sum of squares equation

$$
J(h, \beta)=\sum^{n}\left(y_{i}-x_{i}^{T} \beta-h\left(z_{i}\right)\right)^{2}+\lambda \int_{a}^{b}\left[h^{\prime \prime}\left(z_{i}\right)\right]^{2} d t, \lambda \geq 0
$$

where $\lambda$ is a tüning parameter which controls the tradeoff between goodness of fitting and complexity of the model. It includes the following iterative procedures:

(1) $\lambda$ is the $n \times n$ smooth matrix obtained from univariate cubic spline smoothing, without the parametric terms $x_{i}^{T} \beta$. The transformation of an $n$-vector $\mathrm{z}$ to $\mathrm{S} \lambda$ can be conducted in order of operations

(2) Transforming y to $\tilde{Y}=\left(I-S_{\lambda}\right) Y$, and transforming $X$ to $\tilde{X}=\left(I-S_{\lambda}\right) X$

$$
\beta_{\lambda}=\left(\tilde{X}^{T} \tilde{X}\right)^{-1} \tilde{X}^{T}\left(I-S_{\lambda}\right) Y
$$

(3) Computing

$$
h=\left(\tilde{X}^{T} \tilde{X}\right)^{-1}
$$

The output vector $\tilde{Y}$ is then estimated by

$$
\tilde{Y}=S_{\lambda}+\tilde{X}\left(\tilde{X}^{T} \tilde{X}\right)^{-1}\left(I-S_{\lambda}\right)
$$

(4) Selecting a value for the smoothing parameter $\lambda$ based on the minimum of the generalized cross-validation (GCV) criterion

$$
G C V(\lambda)=\frac{n(Y-\hat{Y})^{T}(Y-\hat{Y})}{\left(n-\operatorname{tr} H_{\lambda}\right)^{2}}
$$

(5) Compute $\operatorname{tr} H_{\lambda}$

$$
\operatorname{tr} H_{\lambda}=\operatorname{tr} S_{\lambda}+\operatorname{tr}\left(\tilde{X}^{T} \tilde{X}\right)^{-1} \tilde{X}^{T}\left(I-S_{\lambda}\right) \tilde{X}
$$

The trace of $S_{\lambda}$ in Equation (7) can be computed using algorithms from spline smoothing ${ }^{15)}$.

\section{Computational results}

As mentioned above, we determined six LRAD measuring for channel contamination, i.e., channel diameter, channel length, distance to (radioactive) source, (radioactivity) strength, wind speed and flux, and obtained corresponding result for alpha particle ionizing current which directly reflects the degree of alpha particle contamination. Thus, the system includes 6 inputs and 1 output. Totally, 400 sampling data were collected, among which 300 samples were used for model training, 80 samples for model testing and the rest 20 samples for prediction. In order to evaluate the effectiveness of the proposed method, two types of errors are adopted: normalized mean squared error (NMSE) and Pearson relative coefficient (PRC), as follows:

$$
N M S E=\frac{\sum_{i=1}^{n}\left(y_{i}-\hat{y}_{i}\right)^{2}}{\sum_{i=1}^{n}\left(y_{i}-\bar{y}_{i}\right)^{2}}, \quad \text { PRC }=\frac{\sum_{i=1}^{n}\left(y_{i}-\bar{y}_{i}\right)\left(\hat{y}_{i}-\overline{\hat{y}}_{i}\right)}{\sqrt{\sum_{i=1}^{n}\left(y_{i}-\bar{y}_{i}\right)^{2}\left(\hat{y}_{i}-\overline{\hat{y}}_{i}\right)^{2}}}
$$


$y_{i}$ is the original value, $\hat{y}_{i}$ is the computational value.

Smaller values of the NMSE indicate smaller deviations between original values and predicted values. The accuracy of the model is evaluated by the PRC. Larger values of PRC (maximum value is 1) correspond to better forecasting performance of the proposed model.

ANN parameters were set as follows: the number of neurons in the hidden layer was the same number of input factor. The learning rate is 0.9 ; the momentum factor is 0.7 ; the iteration times are 1000; the global error is 0.001 . According to the previous literature, there are a variety of methods for ensemble in the past studies. For the purpose of comparison, we have also built other two ensemble forecasting models: simple averaging $^{16)}$, simple MSE $\operatorname{approach}^{17)}$.

Tables 1 and 2 summarize the NMSE and PRC errors for fitting to 300 training samples and 80 testing samples with different models, respectively. These values indicate the learning ability or quality of fitting. The PRC is an efficiency index and indicates that the proposed HRNN ensemble model is deserved to be confident. The results show that the HRNN ensemble model has better fitting performance than those of other ensemble models for the given data.

Table 1 Error comparison of ensemble models for training samples.

\begin{tabular}{ccc}
\hline Method & NMSE error & PRC error \\
\hline Simple Averaging & 0.224 & 0.867 \\
\hline Simple MSE & 0.243 & 0.879 \\
\hline HRNN & 0.062 & 0.963 \\
\hline
\end{tabular}

Table 2 Error comparison of ensemble models for testing samples

\begin{tabular}{ccc}
\hline Method & NMSE error & PRC error \\
\hline Simple Averaging & 0.327 & 0.822 \\
\hline Simple MSE & 0.386 & 0.846 \\
\hline HRNN & 0.081 & 0.947 \\
\hline
\end{tabular}

The more important factor to measure performance of a method is to check its forecasting ability of testing samples. Tables 1 and 2 indicate that the HRNN model has the best generalization ability among three models. Figure 1 shows comparisons of results obtained from the three model and measured data for 20 testing samples. It is found that the proposed HRNN model can reach the best prediction accuracy. Nevertheless, one can see that the HRNN model has a room for improvement for prediction of extreme values. A future work will focus on improving prediction of extreme values. Using the proposed HRNN model, we can depict the picture of alpha particle activity inside a contaminated channel.

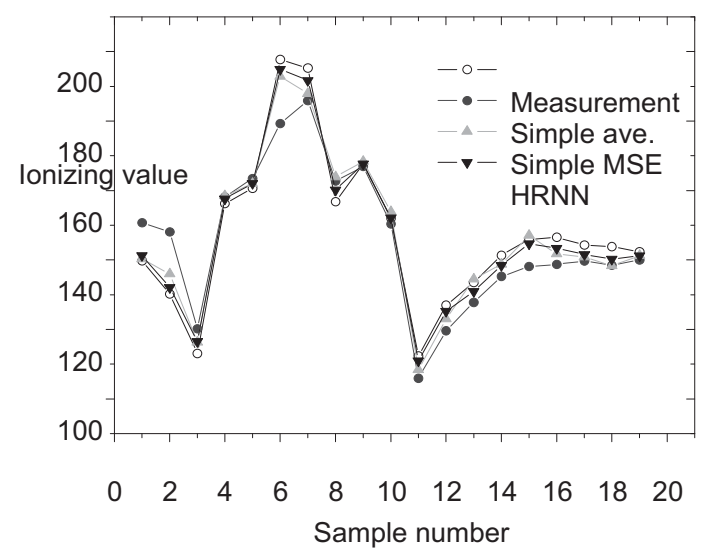

Fig. 1 Comparison between measurement and prediction results of 20 samples with three models.

\section{Conclusion}

This paper introduced a hybrid regression neural network (HRNN) model to predict alpha particles activity inside decommissioned channels for nuclear decommission. The proposed model integrates linear and nonlinear regression models. The computational results indicate that the HRNN ensemble model can be used as an alternative solution for alpha particle activity correction. Once the six parameters are determined, the HRNN model will give an approximate output, thus, an integrated picture of alpha particle activity inside contaminated channels can be obtained. However, the model cannot exactly predict extreme values which would be improved in the future work.

In this article, we used the known source strength and put it in a given position to measure ionizing values in order to check the feasibility of the proposed method. In practice, the distance or source strength is unknown and should be estimated from the ion current. Therefore, how to calculate the distance or source strength from the ion current in reverse is our next step work.

\section{Acknowledgment}

Authors would like to express their sincere thanks to the editor and reviewers for their suggestions to improve the quality of this paper. This work was supported by NSFC (Grant No. 41025015), SKLGP (Grant No. SKLGP2011Z006) and CDUT (Grant Nos. HY0084, HG0092).

\section{References}

1) D.W. MacArthur, K.S. Allander, J.A. Bounds, K.B. Butterfield, Small Long-Range Alpha Detector(LRAD) with Computer Readout, Los Alamos National Laboratory publication LA- 12199-MS, (1991).

2) D.W. MacArthur. Long-range alpha detector (LRAD), LA-UR-91-3398, (1991).

3) D.W. MacArthur. Long-range alpha detector (LRAD), LA-UR-91-3397, (1991).

4) D.W. MacArthur. Long-range alpha detector for contamination monitoring, LA-UR-91-3396, (1991).

5) R.D. Bolton, Radon Monitoring Using Long-Range Alpha Detector-Based Technology, LA-UR-94-3637, (1994). 
6) D.W. MacArthur, K.S.Allander, J.A.Bounds, J.L. MacAtee, "LRAD-Based Alpha-particle Contamination Monitoring of Personnel and Equipment," Nuclear Technology, vol. 102, pp. 270-276 (1993).

7) M.W. Rawool-Sullivan, K.S. Allander, J.A. Bounds, J.E. Koster, D.W. MacArthur, L.L. Sprouse, D. Stout, J.A. Vaccarella, T.Q. Vu, Field study of alpha characterization of a D\&D site using long-range alpha detector, LA-UR-94-3632 (1994).

8) G. Bowden, G. Dandy, H. Maier, "Input determination for neural network models in water resources applications, Part 1. Background and Methodology", Journal of Hydrology, 301, 75-92 (2005).

9) H. Maier, "Input determination for neural network models in water resources applications, Part 2. Case study: Forecasting salinity in a river", Journal of Hydrology, 301, 93-107 (2005)

10) J. Ashu, M. Avadhnam, "Hybrid neural network models for hydrologic time series forecasting", Applied Soft Computing, 7, 585-592 (2007).

11) I. Helland, "PLS regression and statistical mode", Journal of Statistics, 179, 97-114 (1990).
12) Y. Zhou, J. Wu, F. Qin, "Neural network with partial least square prediction model based on SSA and MGF", Proceedings of the 6th World Congress on Intelligent Control and Automation, 2006.

13) D. Kenneth, "Using recursive regression to explore nonlinear relationships and interactions: A tutorial applied to a multicultural education study", Practical Assessment Research and Evaluation, 14(3), 1-13 (2009).

14) M.P. Wand, "Semiparametric regression and graphical models", Centre for Statistical and Survey Methodology, University of Wollongong, Working Paper 18-08, 2008 http://ro.uow.edu.au/cssmwp/15.

15) R.L. Eubank, Spline regression. In: M. G. Schimek (Eds.): Smoothing and regression approaches, computation, and application, Wiley, New York (2000).

16) C.M. Bishop, Neural networks for pattern recognition, Oxford University Press, (1995).

17) A. Krogh, J. Vedelsby, Neural network ensembles, cross validation, and active learning, in: G. Tesauro, D. Touretzky, T. Leen (Eds.), Advances in Neural Information Processing Systems, Vol. 7, MIT Press, Cambridge, MA, 231-238, (1995). 